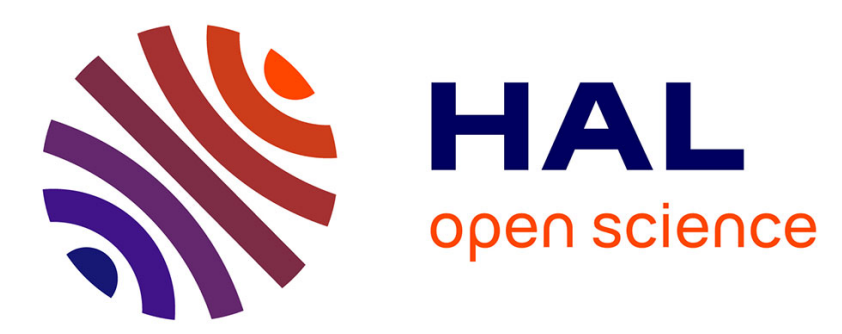

\title{
A hierarchical bayesian dust SED model and its application to the nearby universe
}

\author{
Frédéric Galliano
}

\section{To cite this version:}

Frédéric Galliano. A hierarchical bayesian dust SED model and its application to the nearby universe. Challenges in Panchromatic Modelling with Next Generation Facilities. Conference of the International Astronomical Union, Nov 2018, Osaka, Japan. pp.138-142, 10.1017/S1743921319002394. hal-03047458

\section{HAL Id: hal-03047458 \\ https://hal.science/hal-03047458}

Submitted on 9 Dec 2020

HAL is a multi-disciplinary open access archive for the deposit and dissemination of scientific research documents, whether they are published or not. The documents may come from teaching and research institutions in France or abroad, or from public or private research centers.
L'archive ouverte pluridisciplinaire HAL, est destinée au dépôt et à la diffusion de documents scientifiques de niveau recherche, publiés ou non, émanant des établissements d'enseignement et de recherche français ou étrangers, des laboratoires publics ou privés. 


\title{
A Hierarchical Bayesian Dust SED Model and Its Application to the Nearby Universe
}

\author{
Frédéric Galliano ${ }^{1}$ \\ ${ }^{1}$ AIM, CEA, CNRS, Université Paris-Saclay, Université Paris Diderot, Sorbonne Paris Cité, \\ F-91191 Gif-sur-Yvette, France \\ email: frederic.galliano@cea.fr
}

\begin{abstract}
In this paper, I review several dust evolution studies based on the DustPedia nearby galaxy sample. I first present the dust spectral energy distribution model, implementing a hierarchical Bayesian method, that we have developed. I then discuss the dust evolution trends we have derived among (integrated) and within (resolved) galaxies. In particular, we show that the trend of dust-to-gas ratio with metallicity is clearly non-linear, indicating the need for grain growth in the interstellar medium. Our trend is closer to the one derived with damped Ly $\alpha$ systems than what was suggested by previous studies. We finally demonstrate the universal processing of small amorphous carbon grains by stellar photons.
\end{abstract}

Keywords. methods: statistical, dust, ISM: evolution, galaxies: evolution, infrared: ISM

\section{Introduction}

The properties of interstellar dust (chemical composition, size distribution, etc.) depend on the local environmental conditions, and on the elemental enrichment history of the galaxy (e.g. Galliano et al. 2018). However, the individual processes controlling this evolution (dust production by stars, dust growth in the interstellar medium (ISM), dust destruction by shocks, etc.) are not known accurately enough to unambiguously model the evolution of galaxies. One of the ways to refine our knowledge of these evolutionary processes consists in studying the variations of local observed dust properties, measured via their spectral energy distribution (SED), as a function of the local physical conditions (ultraviolet (UV) field, metallicity, etc.). These trends between dust properties and physical conditions are valuable constraints on the nature and efficiency of the grain evolutionary processes. This leaves us with the task of properly deriving dust parameters and their uncertainties from broadband infrared (IR) observations.

\section{A Hierarchical Bayesian Dust Spectral Energy Distribution Model}

This task of properly deriving dust parameters is known to present several intricate degeneracies and noise-induced false correlations (e.g. Shetty et al. 2009). We have recently developed a model (HerBIE; Galliano 2018) to address this issue.

\subsection{The Microphysical Dust Model}

The microscopic framework of our code is the THEMIS dust model (Jones et al. 2017). This model is consistent with the extinction, depletion patterns and emission (including the Planck data) properties of the diffuse Galactic ISM. THEMIS assumes two grain types (cross-sections from laboratory measurements): (i) amorphous carbons, partially hydrogenated, noted a-C(:H), carrying the aromatic and aliphatic features; and (ii) a$\mathrm{C}(: \mathrm{H})$ coated amorphous silicates with iron inclusions. We parameterize the dust size 

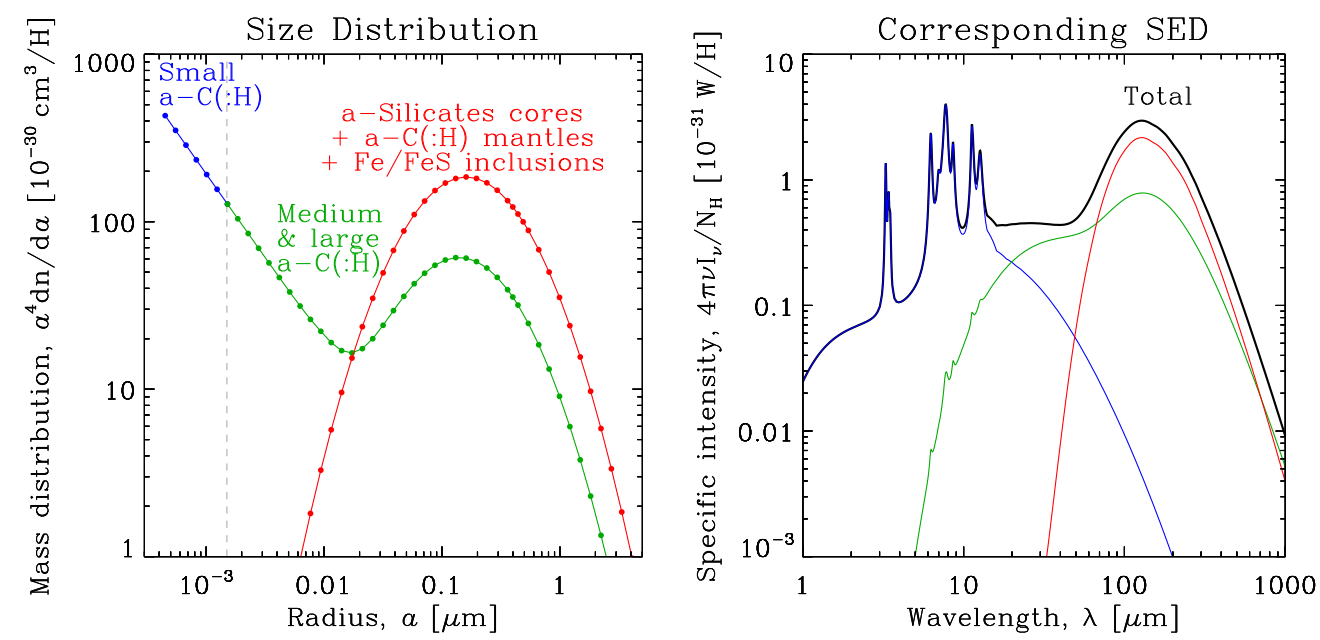

Figure 1. Parameterization of the THEMIS model implemented in HerBIE. The small (medium and large) a-C(:H) have a radius smaller (larger) than $1.5 \mathrm{~nm}$. The color code of the three subcomponents is identical in the two panels.

distribution by scaling the fraction of small a-C(:H), the sub-component carrying the mid-IR features (Fig. 1).

\subsection{The Macroscopical Mixing of Physical Conditions}
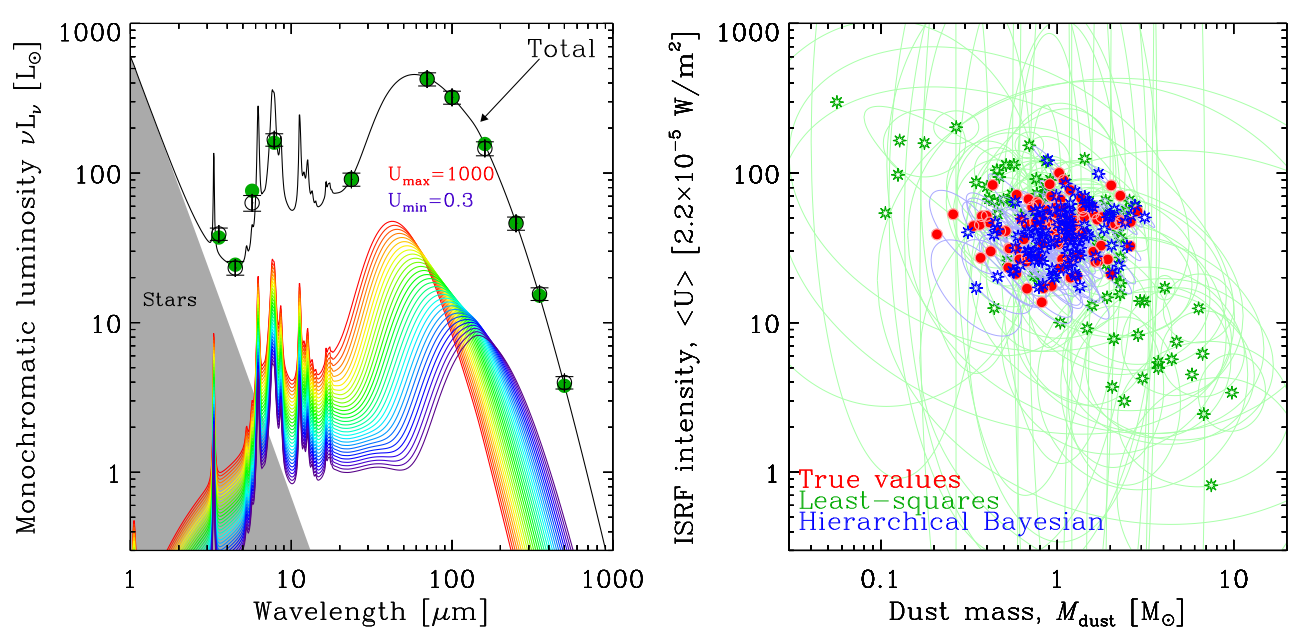

Figure 2. Left: distribution of starlight intensities (color curves) fit to an SED. Right: simulation to demonstrate the efficiency of different methods to fit dust SEDs.

When studying galaxies, we can not avoid the mixing of physical conditions within the observed region. To compensate our ignorance of the particular ISM structure of our sources, we adopt the empirical prescription of Dale et al. (2001), assuming that the distribution of starlight intensities heating the dust, $U$, follows a power-law: $d M_{\text {dust }} \propto$ $U^{-\alpha} d U$ for $U_{\min }<U<U_{\max }$. Thus, the shape of the observed far-IR peak constrains this distribution. This is shown in Fig. 2-left, where the sum of the colored curves is the total dust SED, shown with the black solid line. With this SED model, the main relevant 
parameters, which are weakly dependent on the assumptions we made, are: (i) the dust mass, $M_{\text {dust }}$; (ii) the mean starlight intensity, $\langle U\rangle$; and (iii) the mass fraction of small $\mathrm{a}-\mathrm{C}(: \mathrm{H}), q_{\mathrm{AF}}$.

\subsection{The Statistical Treatment}

To fit this SED model to broadband observations, we have implemented a hierarchical Bayesian (HB) approach (Galliano 2018, for a detailed presentation). In short, HB modelling consists in simultaneously sampling the probability distribution function (PDF) of the model parameters of each individual galaxies, and their statistical distribution over the whole sample. This approach is particularly relevant to solve the numerous noiseinduced false correlations between dust parameters, obtained with standard techniques. Fig. 2-right demonstrates this point, with a simulation. Compared to the true values (red points), the least-squares results (green points with error ellipses) exhibit a false anticorrelation, while the HB results (blue points with error ellipses) allow us to recover the statistical properties of the sample without any bias.

\section{The Studied Nearby Galaxy Sample}

We have applied this model to the near-IR-selected, volume-limited, nearby galaxy sample, DustPedia (Davies et al. 2017). In order to improve our low-metallicity coverage, we included in our analysis the 34 sources of the dwarf galaxy sample (DGS; Madden et al. 2013; Rémy-Ruyer et al. 2013), that were not in DustPedia. In total, our sample contains 813 objects. The photometry comes from Clark et al. (2018) and the total gas mass and metallicities from De Vis et al. (submitted).

\section{Constraints on Dust Evolution}

\subsection{Cosmic Evolution of the Dust Content}

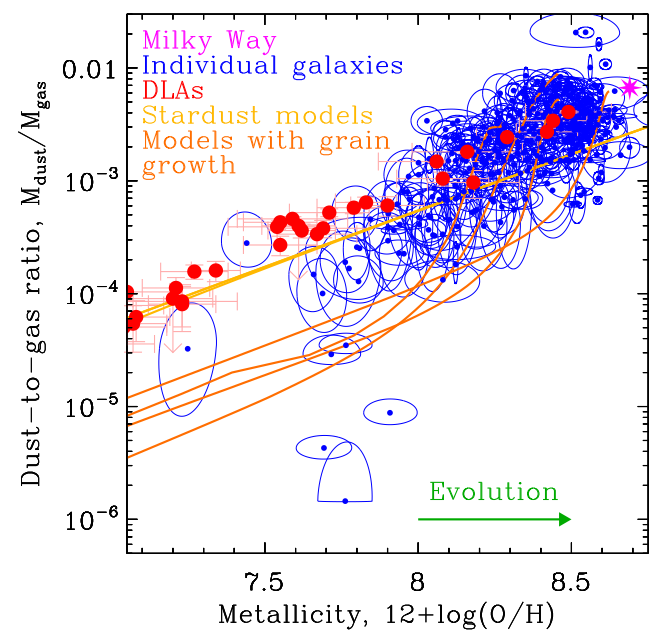

Figure 3. Derived evolution of the dust-to-gas ratio with metallicity for our nearby galaxy sample (blue) and for DLAs (red), compared to dust evolution models (lines).

The most important relation that we can derive from our modelling is the trend of dustto-gas ratio as a function of metallicity. If we consider each galaxy as a snapshot of dust 
evolution at a given stage, then this relation shows how the dust content is built-up from the available heavy elements in the ISM. Fig. 3 shows that this relation from our nearby galaxy sample (blue points with error ellipses; Galliano et al., in prep.) is non-linear. Compared to the dust evolution models of De Vis et al. (2017, yellow and orange lines), it indicates that dust growth in the ISM is a crucial ingredient. Indeed, in models where only stardust contributes to dust production, we are left with linear relation (yellow), inconsistent with the data. The particular care we gave to having an homogenized sample and applying a rigorous statistical treatment to the fitting, allow us to provide the most accurate relation that has been published to date. Interestingly enough, this new trend is in much better agreement with the trend obtained from damped Ly $\alpha$ systems (DLA; De Cia et al. 2016), than the previous trends derived on nearby galaxies (Galliano et al. 2018, for a review).

\subsection{Evolution of the Aromatic Feature Carriers}

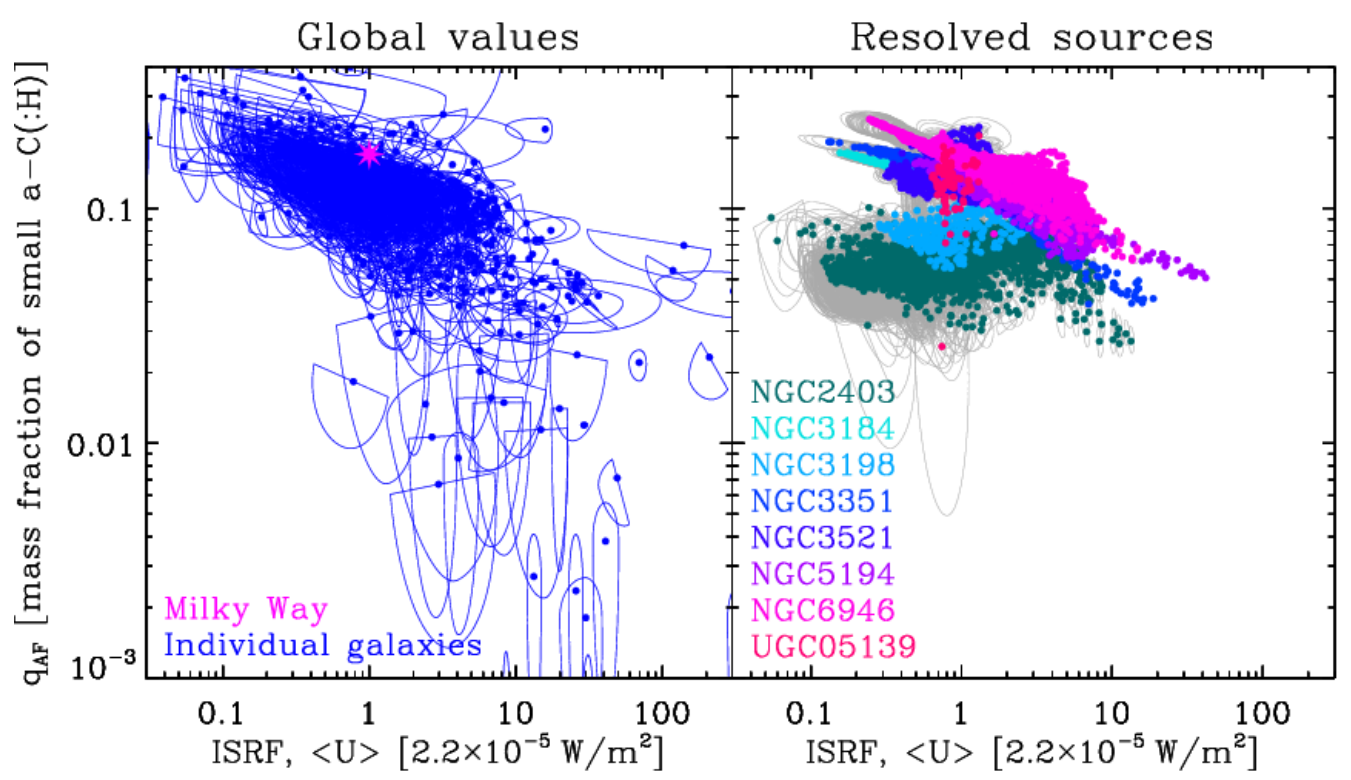

Figure 4. Processing of small a-C(:H) by intense ISRF, among and within galaxies.

Fig. 4 shows the trend between the fraction of small a-C(:H) and the ISRF, among (left) and within (right) galaxies. It clearly confirms that these grains are destroyed by intense radiation fields, as expected.

\section{References}

Clark, C. J. R., Verstocken, S., Bianchi, S., Fritz, J., Viaene, S., Smith, M. W. L., Baes, M., Casasola, V., et al. 2018, A\&3A, 609, A37

Dale, D. A., Helou, G., Contursi, A., Silbermann, N. A., \& Kolhatkar, S. 2001, ApJ, 549, 215

Davies, J. I., Baes, M., Bianchi, S., Jones, A., Madden, S., Xilouris, M., Bocchio, M., Casasola, V., et al. 2017, PASP, 129, 044102

De Cia, A., Ledoux, C., Mattsson, L., Petitjean, P., Srianand, R., Gavignaud, I. \& Jenkins, E. B. 2016, A\&A, 596, A97

De Vis, P., Gomez, H. L., Schofield, S. P., Maddox, S., Dunne, L., Baes, M., Cigan, P., Clark, C. J. R., et al. 2017, MNRAS, 471, 1743 
De Vis, P., Jones, A., Viaene, S., Casasola, V., Clark, C. J. R., Baes, M., Bianchi, S., Cassara, L. P., et al. 2019, A\&A, in press

Galliano, F. 2018, MNRAS, 476, 1445

Galliano, F., De Vis, P., Galametz, M., et al. 2019, in prep.

Galliano, F., Galametz, M., \& Jones, A. P. 2018, ARA $\& A$ A, 56, 673

Jones, A. P., Köhler, M., Ysard, N., Bocchio, M., \& Verstraete, L. 2017, A\&̊A, 602, A46

Madden, S. C., Rémy-Ruyer, A., Galametz, M., Cormier, D., Lebouteiller, V., Galliano, F., Hony, S., Bendo, G. J., et al. 2013, PASP, 125, 600

Rémy-Ruyer, A., Madden, S. C., Galliano, F., Hony, S., Sauvage, M., Bendo, G. J., Roussel, H., Pohlen, M., et al. 2013, A\& A, 557, A95

Shetty, R., Kauffmann, J., Schnee, S., \& Goodman, A. A. 2009, ApJ, 696, 676

\section{Discussion}

Hiroyuki Hirashita: Regarding the relation between small a-C(:H) and metallicity, you said that Seok et al. (2014)'s model has a problem in a scatter caused by star formation histories. Why is the scatter a problem?

FrÉDÉRIC Galliano: My argument was that the small a-C(:H) evolution mechanism proposed by Seok et al. (2014; S14) was interesting, but difficult to test, observationally. I tried overplotting the S14 tracks on my observed trends: they extend beyond the area covered by my data. This could be a mean of constraining the star formation histories of these galaxies. However, S14 model tracks for the dust-to-gas ratio evolution with metallicity, for a similar range of SFHs, cover an area more consistent with the data. It means that, for a given SF timescale (e.g. $0.5 \mathrm{Gyr}$ ), the model will correctly predict the dust-to-gas ratio of some galaxies, but not the fraction of small a-C(:H). It does not mean that the S14 process is wrong, but that there might be other parameters.

Adam Carnall: Are the "individual galaxies" fitted within the BMH?

FRÉDÉRIC GALliano: Yes, the results of the spatially resolved, individual galaxies are from a hierarchical run. In what I presented, there is an independent HB run for the integrated sources, and one independent run for each of the spatially resolved objects. All the physical parameters, as well as the dependencies (gas and stellar masses, metallicity, etc.) are in the hierarchical model.

Adam Carnall: How do you sample from the posterior distribution of the BMH?

FrÉDÉRIC Galliano: I performed Gibbs sampling on each of the parameters and hyperparameters. The sampler is a multithreaded Fortran code I developed. I ended up developing my own sampler, as all the publicly available samplers I tested were not adapted for high dimensionality. For instance, in some of the galaxies I presented, there are almost 100, 000 pixels. With a seven parameter model, I have to sample a posterior in $\mathrm{a} \simeq 700,000$ dimension parameter space...

Adam Carnall: Can you construct conditional distributions for all your parameters?

FrÉdÉRIC GALliano: Yes, I can construct conditional posteriors for each of the model parameters and dependencies.

HeLEN KIM: For your sample of 875 galaxies, what criteria did you use to determine they had sufficient IR data? 
FrÉdÉric Galliano: Among the 3000, NIR and volume-limited selected, DustPedia galaxies, the 875 objects we modeled the dust SEDs were those which had been observed with Herschel and had a size larger than 1 arcminute.

Fumi Egusa: How do you treat ionization state of dust grains in the model?

FRÉDÉRIC GALLIANO: There is currently no account of the effects of grain charging in the dust model I used (THEMIS; Jones et al. 2017). Other dust models, such as Draine \& Li (2007), include a PAH component, constituted of neutral and charged molecules. The ratio of this mixture can be varied in order to empirically change the aromatic feature spectrum and obtain a better fit. However, there is, to my knowledge, no reliable constraints on the effect of charge of small a-C $(: \mathrm{H})$, from laboratory data. In the modeling I presented, I am able to adapt the aromatic feature spectrum in order to fit the complexity of the data, by dividing the size distribution of small a- $\mathrm{C}(: \mathrm{H})$ into two bins, whose weight can be varied. 